\title{
TSC1-mTOR signaling determines the differentiation of islet cells
}

\author{
Li Ding1,,", Yue Yin1,, Lingling Han1,", Yin Li1, Jing Zhao' and Weizhen Zhang1,2 \\ 1Department of Physiology and Pathophysiology, Peking University Health Science Center, and Key Laboratory of \\ Molecular Cardiovascular Science, Ministry of Education, Beijing, China \\ 2Department of Surgery, University of Michigan Medical Center, Ann Arbor, Michigan, USA \\ *(L Ding, Y Yin and L Han contributed equally to this work)
}

Correspondence

should be addressed

to W Zhang

Email

weizhenzhang@bjmu.edu.cn

\begin{abstract}
Neurogenin3-driven deletion of tuberous sclerosis complex 1 (Tsc1) activated mechanistic target of rapamycin complex 1 (mTORC1) measured by the upregulation of mTOR and S6 phosphorylation in islet cells. Neurogenin3-Tsc1-/- mice demonstrated a significant increase in average islet size and mean area of individual islet cell. Insulin mRNA and plasma insulin levels increased significantly after weaning. Glucagon mRNA and plasma levels increased in neonate followed by modest reduction in adult. Somatostatin mRNA and plasma levels markedly increased. Neurogenin3-Tsc1-/- mice fed standard chow demonstrated a significant improvement in glucose tolerance and no alteration in insulin sensitivity. In Neurogenin3-Tsc1-/- mice fed 45\% high-fat diets, both glucose tolerance and insulin sensitivity were significantly impaired. Rapamycin reversed the activation of mTORC1, attenuated $\beta$ cells hypertrophy and abolished the improvement of glucose tolerance. TSC1-mTORC1 signaling plays an important role in the development of pancreatic endocrine cells and in the regulation of glucose metabolism.
\end{abstract}

\author{
Key Words \\ - mechanistic target of \\ rapamycin complex 1 \\ - differentiation and \\ development \\ - diabetes \\ - glucose metabolism \\ $\checkmark$ insulin
}

\section{Introduction}

Pancreatic islet contains four major types of endocrine cells, including $\alpha, \beta, \delta$ and PP cells, which secret glucagon, insulin, somatostatin and pancreatic polypeptide, respectively. All types of pancreatic endocrine cells arise from the pancreatic endocrine progenitor cells during the embryonic development period under the control of many transcriptional regulators including neurogenin3 (Ngn3). Activation of neurogenin3 expression in the pancreatic and duodenal homeobox 1 (PDX1)-positive precursors triggers the specific gene regulatory networks that define the endocrine program (Gradwohl et al. 2000, Gu et al. 2002). On the other hand, $\mathrm{Ngn} 3$ deficiency impairs the development of islets (Gradwohl et al. 2000). All these data suggest that Ngn3 plays an essential role in the transition from a multipotent pancreatic progenitor toward an endocrine precursor.
Emerging evidence suggests that adaptive response to environmental condition exists during organism development (Holness \& Sugden 1999). In humans and other mammals, maternal undernutrition or stress during gestation results in small offspring with permanently altered metabolism and tissue composition. Maternal nutrition influences both islet development and insulin secretion. Maternal protein or caloric restriction decreases $\beta$ cell mass and insulin content, as well as diminishes insulin secretory response (Snoeck et al. 1990, Duvillie et al. 1997). Consequently, combination of protein restriction during embryonic period with high-fat diet (HFD) in later adult period significantly impairs the insulin sensitivity relative to the control animals fed HFD. This evidence suggests that nutritional supply during embryonic period influences

Published by Bioscientifica Ltd 
the development of islet endocrine cells. The molecular mechanism underlying such response remains unknown.

The mechanistic target of rapamycin (mTOR) is a highly conserved serine-threonine kinase, which has been reported to serve as a fuel sensor (Wullschleger et al. 2006, $\mathrm{Xu}$ et al. 2009). There exist two mTOR complexes: mTOR complex 1 (mTORC1) and mTOR complex 2 (mTORC2). mTORC1 is responsible for nutrient-sensing functions and is composed of mTOR, G protein-subunit-like protein and raptor. mTORC2 phosphorylates Akt protein kinase B and contains mTOR and rictor (Sarbassov et al. 2005). mTORC1 has been demonstrated to coordinate the intracellular energy levels with cell growth (Inoki et al. 2003, 2006) and the organism energy supply with food intake (Xu et al. 2009). It is currently unknown whether mTORC1 can coordinate the embryonic energy status with the development of islet cells. Previous study has shown that administration of leucine, an agonist for mTORC1 signaling pathway, to pregnant female mice causes weight gain, hyperglycemia, hypoinsulinemia and reduction of islet mass in offspring (Rachdi et al. 2012). In contrast, activation of the mTORC1 signaling pathway in the mature $\beta$ cells increases $\beta$ cell mass and size, which is associated with improved glycemic control and insulin production (Mori et al. 2009). Consistently, a recent study suggests that mTORC 1 influences $\beta$ cell proliferation by regulating the synthesis and stability of cyclin D2, a cell cycle protein (Balcazar et al. 2009). To further explore the role of mTORC1 signaling in the differentiation and development of pancreatic endocrine cells, we generated the transgenic mice, Ngn3-Tsc1-/mice, in which the tuberous sclerosis complex 1 (Tsc1) gene was specifically deleted driven by Ngn3 promoter. We report here that deletion of Tsc1 gene activates the mTORC1 signaling in the pancreatic islet cells of Ngn3-Tsc1-/- mice. Increase of mTORC1 activity in pancreatic islet cells during development is associated with $\beta$ cell hypertrophy, hyperinsulinemia and improved glucose tolerance in mice.

\section{Methods}

\section{Materials}

Rabbit anti-phospho-mTOR (ser2448), rabbit antimTOR, mouse anti-TSC1 and rabbit anti-phospho-S6 (ser235, 236) (species cross-reactivity: human, mouse and rat) were purchased from Cell Signaling Technology. Rapamycin and the following antibodies were from Santa Cruz Biotechnology: goat anti-insulin A (C-12) (species cross-reactivity: human, mouse and rat), goat anti-glucagon (N-17) (species cross-reactivity: human, mouse and rat), rabbit anti-somatostatin (FL-116) (species cross-reactivity: human, mouse and rat), goat anti-rabbit fluorescein isothiocyanate (FITC)-conjugated IgG, donkey anti-goat FITC-conjugated IgG, donkey anti-goat Texas Red-conjugated IgG, goat anti-mouse Texas Red-conjugated IgG and chicken anti-rabbit FITC-conjugated IgG. IRDye-conjugated affinity-purified anti-rabbit, anti-mouse IgGs were obtained from Rockland (Gilbertsville, PA, USA). Dimethyl sulfoxide and aprotinin were purchased from Sigma Chemical Co. and Amersham Biosciences, respectively.

\section{Animals and animal care}

Ngn3-Cre mice that express the Cre recombinase gene under the control of the Ngn3 gene promoter, as well as Tsc1 ${ }^{\text {loxP } / l o x P}$ mice, in which exons 17 and 18 of the Tsc 1 gene are flanked by loxP sites by homologous recombination, were purchased from the Jackson Laboratory. The Negn3-Tsc1-/- mice were generated by breeding Tsc1 10xP $/$ oxP mice with Ngn3-Cre mice as described previously (Ding et al. 2014, Xu et al. 2015). Control experiments used littermate Tsc1 ${ }^{\text {loxp } / o x P}$ animals. Deletion of the Tsc1 gene was validated by the absence of its protein, and the increased phosphorylation of mTOR and S6, the downstream signaling molecule of mTOR, in pancreas tissues. Mice were housed on a $12-12 \mathrm{~h}$ light-darkness cycle. Normal chow and water were available ad libitum. Where indicated, 6-week-old animals were fed with $45 \%$ HFD (D12451; Research Diets, New Brunswick, NJ, USA) for 8 weeks. Control diets used D12450H from Research Diets Inc.

\section{Genotyping}

Genomic DNA was extracted from tail cuttings. Polymerase chain reactions were performed to test for the presence of the Tsc1 and/or the deletion of its exons 17 and 18 using LoxP primers, and Cre constructs using cre-specific primers, respectively (Ding et al. 2014, Xu et al. 2015).

\section{Western blot analysis}

Tissues extracts were immunoblotted with antibodies against pmTOR (ser2448) (1:1000 dilution), mTOR (1:1000 dilution), TSC1 (1:600 dilution), S6 (1:1000 dilution), pS6 (1:1000 dilution) and $\beta$-actin

Published by Bioscientifica Ltd 
(1:1000 dilution) as described previously (Mori et al. 2009, Xu et al. 2009, 2015). Briefly, pancreatic tissues were isolated and homogenized in RIPA lysis buffer containing Protease Inhibitor Cocktail. Proteins were subjected to SDS-PAGE with a $10 \%$ running gel, and then transferred to a polyvinylidene fluoride membrane. Membranes were incubated for $1 \mathrm{~h}$ at room temperature with $5 \%$ fat-free milk in Tris-buffered saline containing Tween 20, followed by incubation overnight at $4^{\circ} \mathrm{C}$ with primary antibodies. Specific reaction was detected using IRDye-conjugated second antibody and visualized using the Odyssey infrared imaging system (LI-COR Biosciences, Lincoln, NE, USA).

\section{Immunostaining}

Mice were deeply anesthetized using sodium pentobarbital $(40 \mathrm{mg} / \mathrm{kg}$, i.p.), perfused transcardially with $20 \mathrm{~mL} 0.1 \mathrm{M}$ PBS (pH 7.4), followed by $20 \mathrm{~mL}$ $4 \%$ paraformaldehyde in PBS. Pancreas was quickly removed and fixed in 4\% paraformaldehyde. Pancreas was sectioned perpendicular to its longitudinal axis into parallel slices of approximately $1 \mathrm{~mm}$ thickness, with the first cut positioned randomly within an interval of $1 \mathrm{~mm}$ length at the splenic end of the pancreas. Tissue slices were embedded in paraffin and sectioned at $6 \mu \mathrm{m}$. Tissue sections were de-paraffinized and hydrated by graded washes with xylene and ethanol. Antigen retrieval was accomplished by boiling in $10 \mathrm{mM}$ sodium citrate acid buffer ( $\mathrm{pH}$ 6.0) for $15 \mathrm{~min}$. Endogenous peroxidase activity was blocked by incubation of slides in $3 \% \mathrm{H}_{2} \mathrm{O}_{2}$ for $10 \mathrm{~min}$. Nonspecific binding was blocked with $1 \%$ bovine serum albumin. Sections were then incubated with primary antibodies overnight at $4^{\circ} \mathrm{C}$. For immunohistochemistry, secondary antibody staining was performed with a poly-HRP detection system for rabbit or mouse primary antibody (polink-2 plus HRP, Golden Bridge International, USA) for $1 \mathrm{~h}$ at room temperature. Immunoreactivity was detected using diaminobenzidine substrate (peroxide substrate kit, SK-4100; Vector Laboratories, Beijing, China) for 2-5 min. Slides were then counterstained with Mayer's Hematoxylin before dehydration and mounting. For immunofluorescence, secondary antibodies used included chicken anti-rabbit FITC-conjugated IgG (1:100), goat anti-mouse Texas Red-conjugated IgG (1:100 dilution), goat anti-rabbit FITC-conjugated IgG (1:100 dilution), donkey anti-goat FITC-conjugated IgG (1:100 dilution) and donkey anti-goat Texas Red-conjugated IgG (1:100 dilution). Pancreatic tissues were incubated with secondary antibody for $1 \mathrm{~h}$ at room temperature. Fluorescent signals were observed, and photomicrographs were taken under a confocal laser-scanning microscope (Leica). Controls included substituting primary antibodies with relevant IgGs.

\section{Extraction of RNA and quantitative real-time PCR analysis}

Total pancreatic RNA was isolated using the TRIzol reagent (Invitrogen). Reverse transcription and quantitative real-time PCR were performed as previously described (Li et al. 2008, Xu et al. 2009). PCRs were performed in duplicate, and each experiment was repeated three to five times. Primers used in this study are the following:

Mouse $\beta$-actin ATCTGGCACCACACCTTC AGCCA GGTCCAGACGCA

Mouse TSC1 WT CCTTCACAGACAGTCAAACGA ACTGTCTTCCTCAGAAGCCAGAT

Mouse TSC1 KO CGGCTCTGGAGGAACACAAT T GGCTATGCAGTTGGGTCAC

Mouse Insulin CCTGCCCAGGCTTTTGTCAA CTCCA GTGCCAAGTCTGAA

Mouse Glucagon ACATCTCGTGCCAGTCACTT CG TTGGGTTACACAATGCT

Mouse Somatostatin CCCAGACTCCGTCAGTTTCT ACTTGGCCAGTTCCTGTTTC

\section{Measurement of plasma hormones}

After anesthesia with sodium pentobarbital, blood samples from animals fed ad libitum were transcardially collected in the presence of aprotinin $(2 \mu \mathrm{g} / \mathrm{mL})$ and EDTA $(1 \mathrm{mg} / \mathrm{mL})$. Plasma was harvested and stored at $-70^{\circ} \mathrm{C}$ before use. Levels of plasma insulin, glucagon and somatostatin were measured using distinct rat/mouse enzyme-linked immunosorbent assay and radioimmunoassay (Millipore, Cat.\# EZRMI-13K for insulin, Cat.\# GL-32K for glucagon; Phoenix Pharmaceuticals, RK-060-03 for somatostatin).

\section{Oral glucose tolerance and insulin sensitivity tests}

Oral glucose tolerance tests (OGTT) were performed on mice fasted for $6 \mathrm{~h}(0800 \mathrm{~h}$ to $1400 \mathrm{~h})$. Blood glucose levels were measured at $0,15,30,60,90$ and $120 \mathrm{~min}$ after oral administration of glucose $(2 \mathrm{~g} / \mathrm{kg}$ body weight). For insulin tolerance tests, mice were fasted for $4 \mathrm{~h}(0800 \mathrm{~h}$ to $1200 \mathrm{~h}$ ) and injected with $1 \mathrm{IU} / \mathrm{kg}$ body weight of human insulin. Blood glucose levels were determined by use of a

Published by Bioscientifica Ltd. 
Freestyle Brand Glucometer (Roche) with blood collected from the tail vein.

\section{Glucose clamp studies}

Two-hour hyperglycemic clamps were performed in either WT or TN mice fasted for $4 \mathrm{~h}$ following the instruction of National Institute of Diabetes and Digestive and Kidney Diseases (NIDDK). Briefly, twenty percent dextrose was infused starting $5 \mathrm{~min}$ after the insulin infusion to clamp glycemia at $15 \mathrm{mM}$. Plasma glucose levels were measured at 5-min intervals and maintained at $15 \mathrm{mM}$ by an adjustable infusion of $20 \%$ dextrose. Glucose infusion rate (GIR) was recorded at 5-min intervals.

\section{Rapamycin treatment}

Pregnant mice were randomly divided into two groups. At the near-term pregnancy, mice were injected intramuscularly with rapamycin ( $1 \mathrm{mg} / \mathrm{kg}$ body weight) or vehicle every other day for 7 days before the birth of pups. Rapamycin at the same dose was administrated to
A

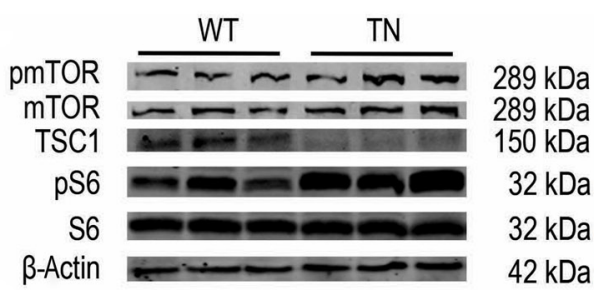

B

WT
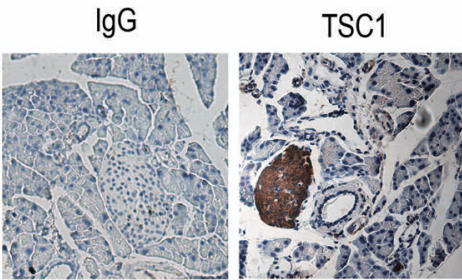

TN

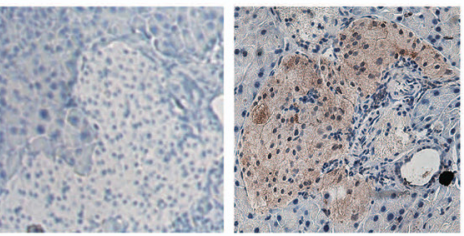

C

$\lg G$
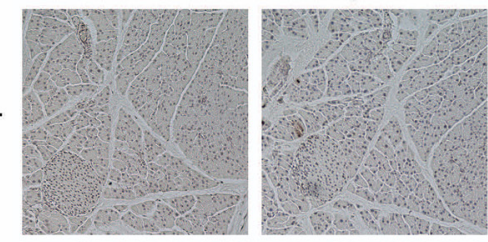

TN

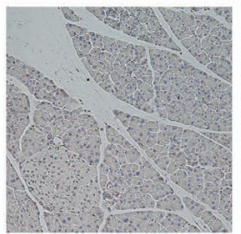

D
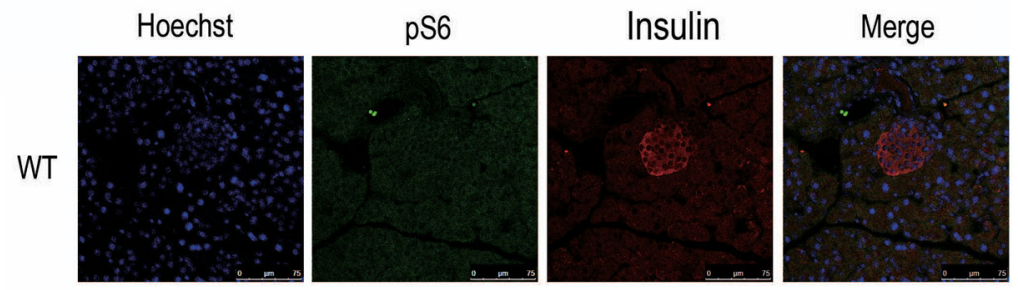

TN
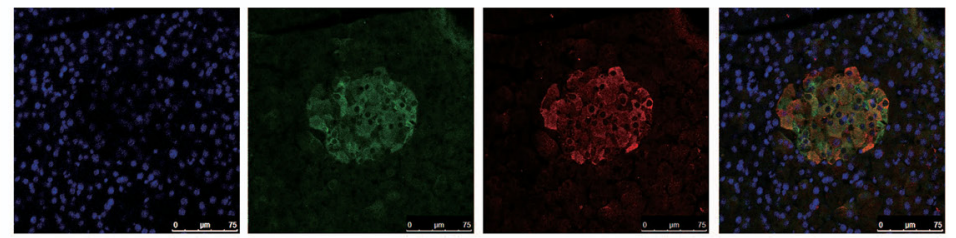

Hoechst

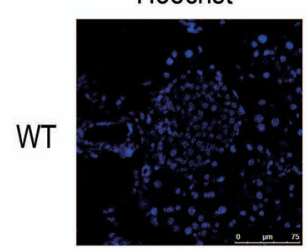

pS6

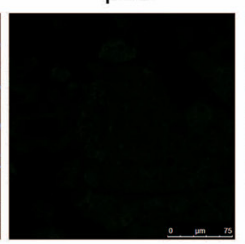

Glucagon
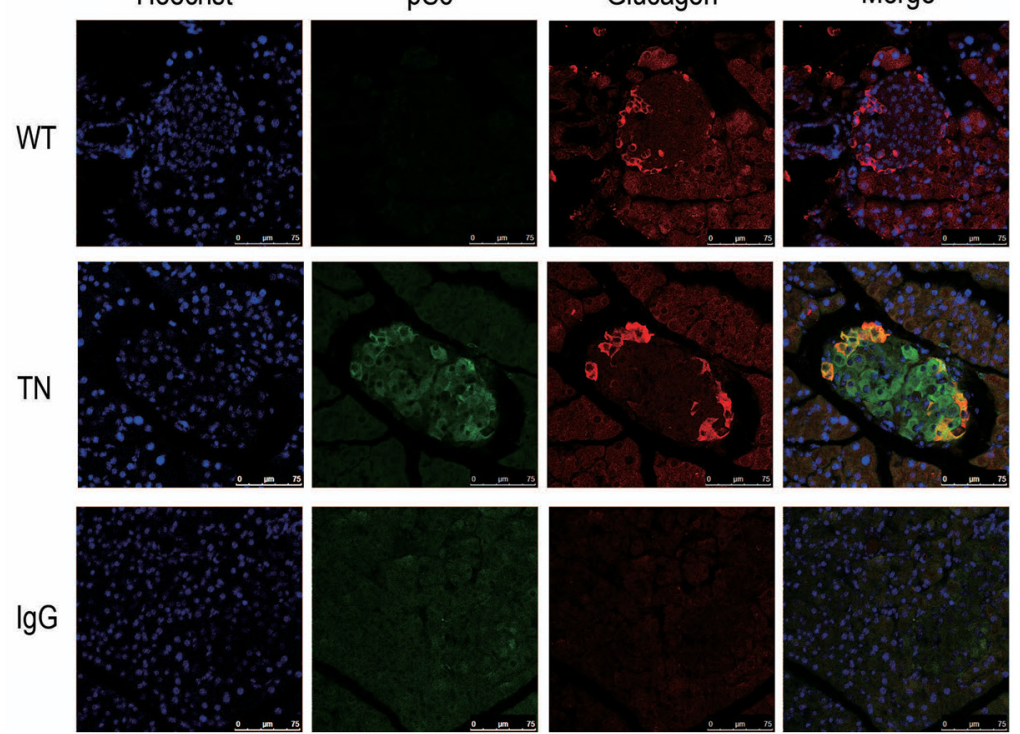

Figure 1

Deletion of TSC1 and activation of mTOR signaling in the pancreas of Ngn3-Tsc1-/- transgenic mice. (A) Representative Western blot TSC1 and phospho-S6 (pS6) in pancreatic tissue were examined using specific antibodies. $n=3$. (B) TSC1 immunoreactivity and (C) pS6 immunoreactivity. Shown are the representative of pancreas from wild-type (WT) and Ngn3-TsC1-I- (TN) mice, respectively. (D) Co-localization of pS6 and insulin or glucagon. Double-labeling of the pancreatic sections from WT and TN mice were performed with pS6 (green) and insulin (red, upper panel) or glucagon (red, lower panel) antibodies. Nuclei were counter-stained with Hoechst 33342 fluorescent stain (blue).

$$
\begin{aligned}
& \text { http://joe.endocrinology-journals.org } \\
& \text { DOI: } 10.1530 / \text { JOE-16-0276 }
\end{aligned}
$$

(c) 2017 Society for Endocrinology Printed in Great Britain

Published by Bioscientifica Ltd 
mother mice continuously until weaning, and then given to offspring for another 4 weeks at the same dose. Plasma and pancreatic tissues were harvested for qPCR, and immunohistochemical analysis was performed $12 \mathrm{~h}$ after the last dose of rapamycin.

\section{Statistical analysis}

Data were expressed as mean \pm S.E.M. and analyzed by repeated-measures analysis of variance, one-way ANOVA, Student-Newman-Keuls test (comparisons between multiple groups), unpaired Student's- $t$ test (between two groups) as appropriate. A value of $P<0.05$ denotes the statistical significance.

\section{Results}

\section{Activation of mTOR signaling in islet cells of Ngn3-Tsc1-/- mice}

By crossbreeding the Nng3-Cre mice with Tsc1loxp mice, we have generated a mouse strain Nng3-Tsc1-/- in which Tsc1 gene is deleted under the control of Ngn 3 promoter. Analysis of pancreatic TSC1 and its related mTORC1 signaling molecules validated the deletion of Tsc1 gene in the pancreatic islet cells. As shown in Fig. 1A and B,
TSC1 was detected at a relative high level in the pancreatic islet cells of wild-type littermates (WT), whereas Nng3-Tsc1-/- (TN) mice demonstrated a significant reduction in levels of TSC1 immunoreactivity. As TSC1 is an inhibitor of mTORC1, we next examined the mTORC1 activity in the pancreas. Phosphorylation of mTOR and S6 ribosomal protein (pS6), the downstream target of mTORC1, was significantly increased in pancreas of Ngn3-Tsc1-/- (TN) mice relative to control wild-type littermates (WT) (Fig. 1A and C).

Neurogenin3 in the pancreatic progenitor cells is often considered as the master transcriptional factor required for the determination of the pancreatic endocrine destiny. To confirm whether the mTORC1 signaling is specifically activated in pancreatic endocrine cells, double immunofluorescent staining was performed to co-localize the expression of insulin or glucagon with pS6 in the pancreas of Ngn3-Tsc1-/- and WT mice. As shown in Fig. 1D, antibody recognizing pS6 showed a strong immunoreactivity in cells stained positive for insulin or glucagon in Ngn3-Tsc1-/- mice, whereas no obvious pS6 signal was detected in WT mice. Control antibodies produced no positive signal. These data indicate that deletion of Tsc1 gene results in the specific activation of mTORC1 signaling in the pancreatic endocrine cells of Ngn3-Tsc1-/- (TN) mice. Thus, we have
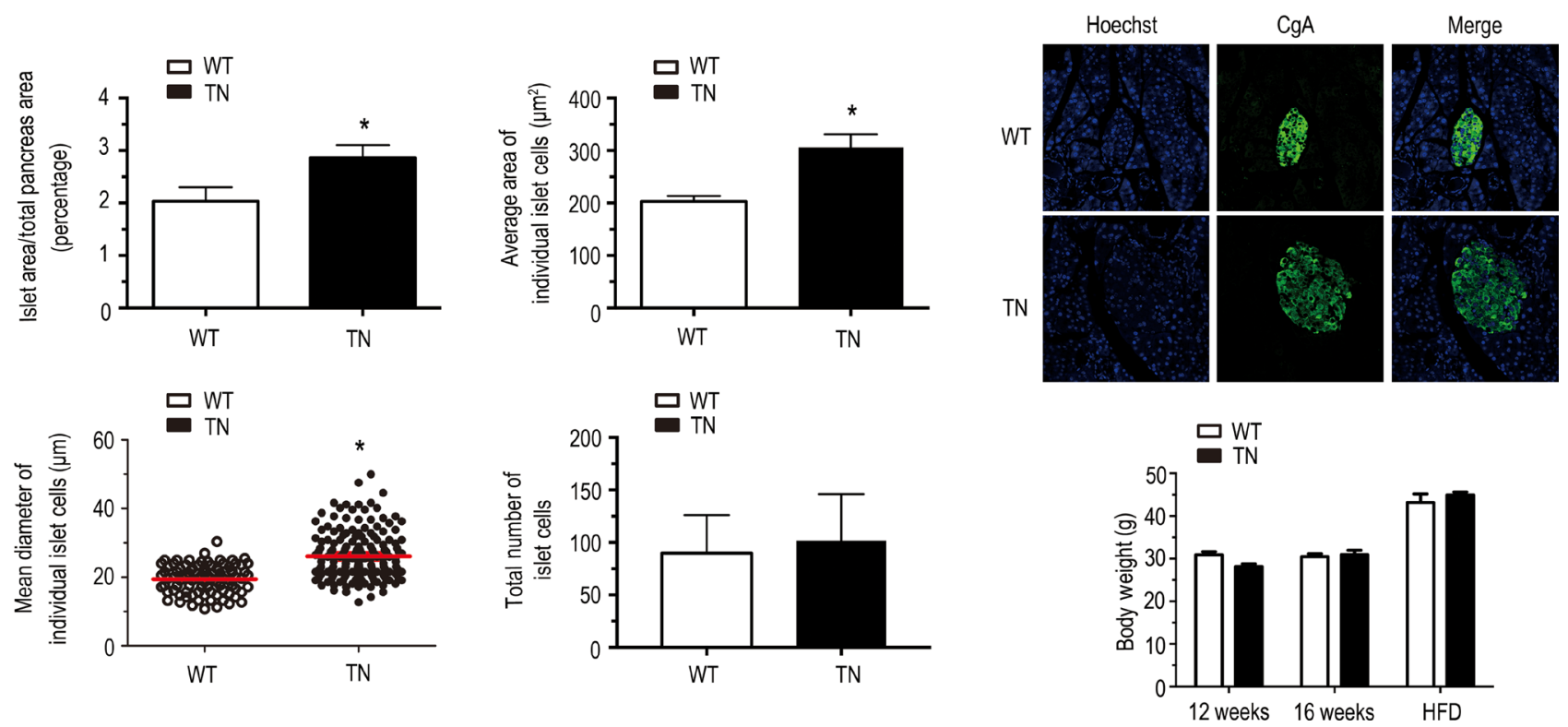

Figure 2

Islet hypertrophy in Ngn3-Tsc1-I- mice. Mean size of islets, average area and diameter of individual islet cell and total number of islet cells were measured in pancreatic sections stained with chromogranin A antibody (CgA) using the ImageJ software. Five tissue sections, each $75 \mu \mathrm{m}$ apart, and five low magnification (10x objective) views of each section were analyzed for each animal using the Leica microscope DMLFS/A. Results were expressed as mean \pm S.E.M. * denotes $P<0.05 . n=7$. 
A

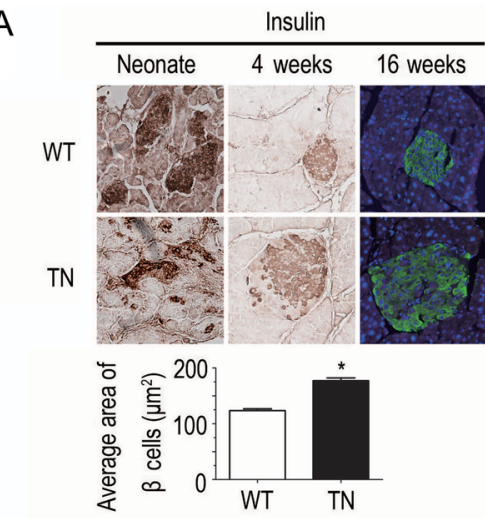

B

WT

TN
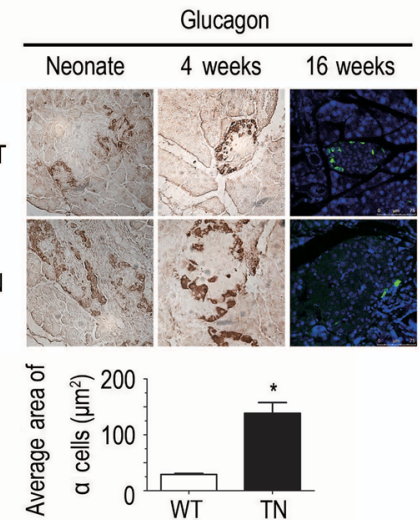

D
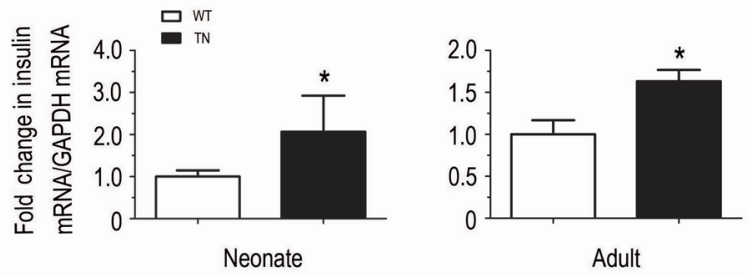

E
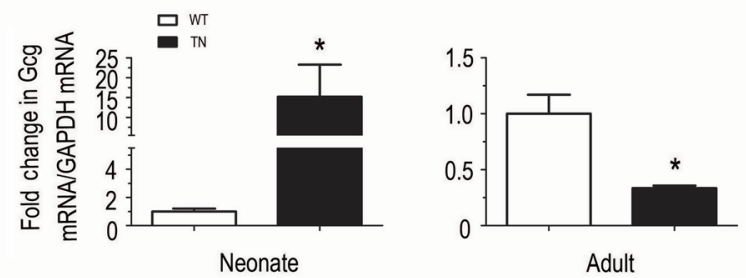

C

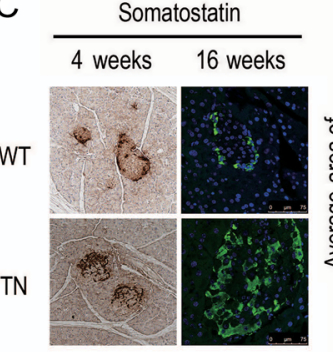

G

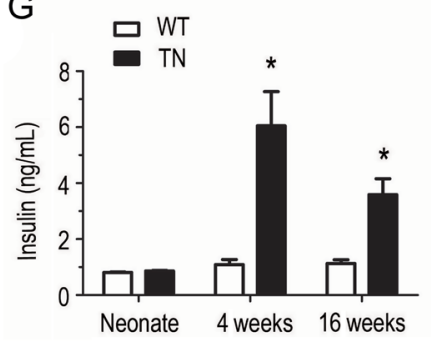

$\mathrm{H}$

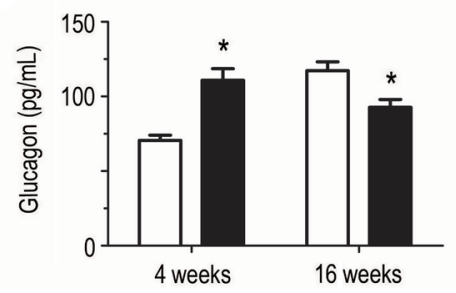

F
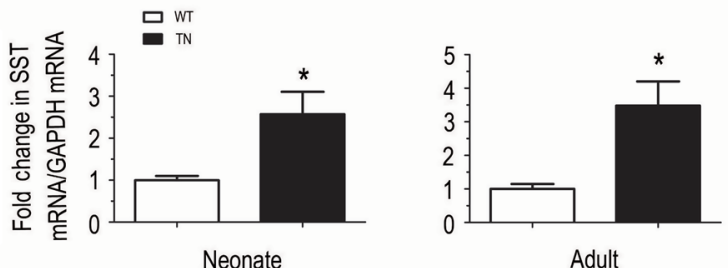

I

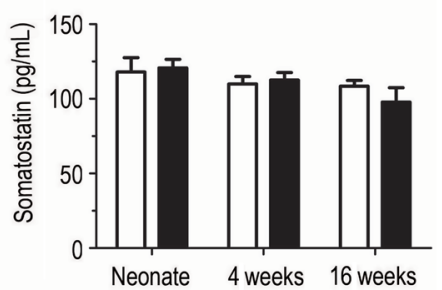

Figure 3

Changes in insulin, glucagon and somatostatin in Ngn3-Tsc1-/- mice. Pancreatic sections of wild-type littermates (WT) and Ngn3-Tsc1-/- (TN) mice were stained with antibodies against insulin (A), glucagon (B) and somatostatin (C), respectively. Real-time PCR quantification of pancreatic insulin (D),

glucagon (E) and somatostatin (F) from neonate and adult WT and TN mice were examined. Serum levels of insulin (G), glucagon (H) and somatostatin (I) from WT and TN mice were measured and expressed as mean \pm S.E.M. * denotes $P<0.05$ vs WT mice. $n=5$. 
successfully generated a mouse strain with pancreatic deletion of TSC1 and subsequent activation of mTORC1 signaling in islet cells.

\section{Islet hypertrophy in Ngn3-TSC1-/- mice}

Given the apparent enlargement of islets observed in the insulin staining (Fig. 1D), we completed a thorough examination on the pancreatic islets of Ngn3-Tsc1-/mice. Chromogranin A $(\mathrm{CgA})$, which stains positive in virtually all endocrine cells within islets, was used to highlight the islet cells. Relative islet mass was calculated as the percentage of islet area over total pancreatic area. As shown in Fig. 2, average islet mass of Ngn3-Tsc1-/mice increased significantly relative to the control littermates (Fig. 2). Mean islet mass was $2.87 \pm 0.24 \%$ for
Ngn3-Tsc1-/- mice vs $2.04 \pm 0.27 \%$ for wild-type littermates $(P<0.01)$. Islet cell hypertrophy accounts for the increase in islet size. Mean area of individual islet cell increased significantly from $203.2 \pm 10.33 \mu 3^{2}$ in wild-type animals to $305.9 \pm 25.32 \mu 2^{2}$ in Ngn3-Tsc1-/mice $(P<0.01)$. Similar increase in the mean diameter of islet cells was also observed $(19.44 \pm 0.45$ vs $26.06 \pm 0.53 \mu 3$; $P<0.0001)$. In contrast, total number of islet cells demonstrated no alteration (Fig. 2). Furthermore, no significant differences was detected in the proportion of BrdU-positive nuclei or TUNEL-positive nuclei in the islets between the Ngn3-Tsc1-/- and wild-type mice at 8 weeks of age (data not shown). Therefore, deletion of TSC1 and subsequent activation of mTORC1 signaling increased cell size rather than proliferation of islet cells, indicating the islet cell hypertrophy in Ngn3-Tsc1-/- mice.
A

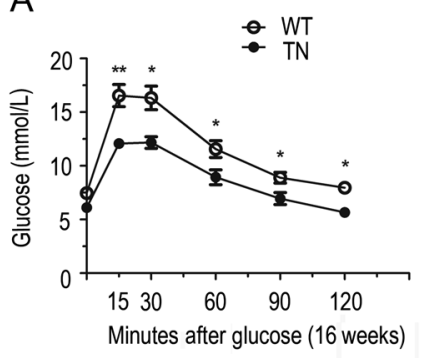

C

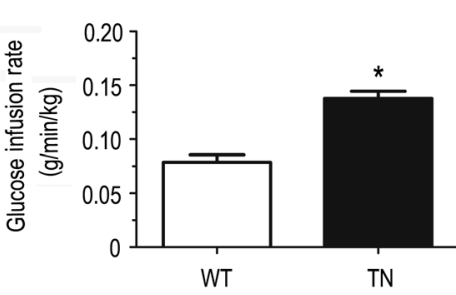

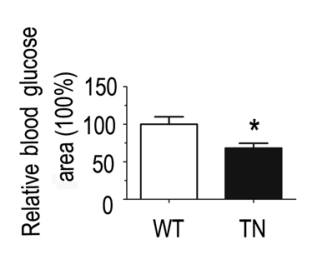

B

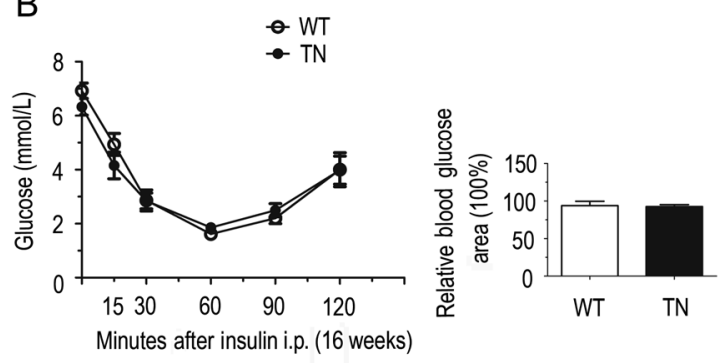

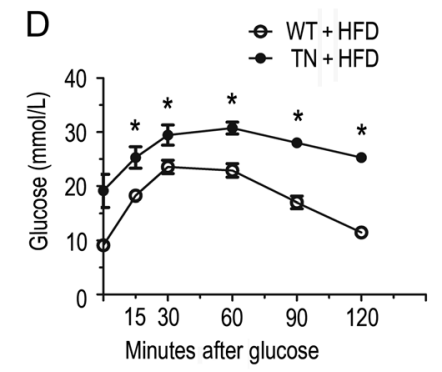

$\mathrm{F}$
E

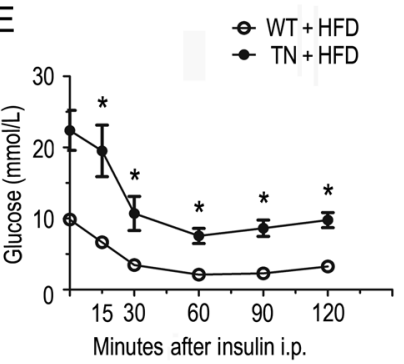

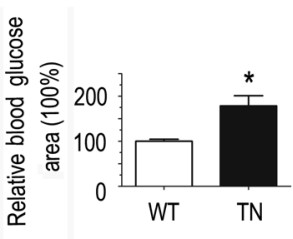
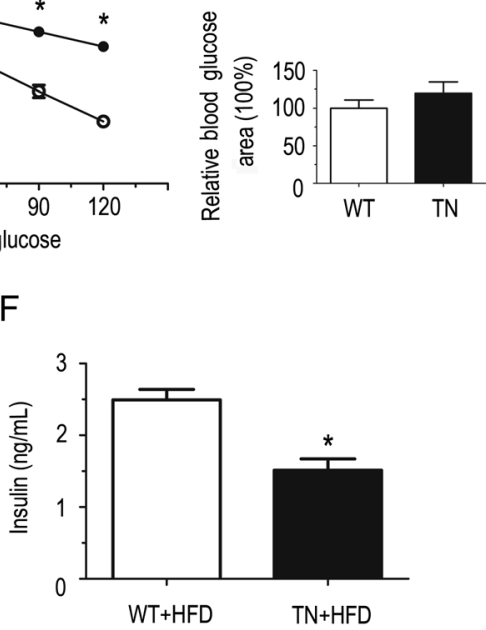

\section{Figure 4}

Alteration in glucose tolerance and insulin sensitivity in Ngn3-Tsc1-/- mice. (A) Blood glucose levels of wild-type littermates (WT) and Ngn3-Tsc1-/- (TN) mice fed NCD after oral administration of glucose ( $2 \mathrm{~g} / \mathrm{kg}$ body weight). (B) Blood glucose levels of WT and TN mice fed NCD after intraperitoneal injection of insulin (1 IU/kg body weight). (C) Glucose infusion rate (GIR) was examined by hyperglycemic clamps in WT and TN mice. (D) Blood glucose levels of WT and TN mice fed HFD after oral administration of glucose ( $2 \mathrm{~g} / \mathrm{kg}$ body weight). (E) Blood glucose levels of WT and TN mice fed HFD after intraperitoneal injection of insulin (1 IU/kg body weight). (F) Serum levels of insulin in WT and TN mice fed HFD. Results were expressed as mean \pm S.E.M. *denotes $P<0.05$ vs wild-type mice. $n=6$.

http://joe.endocrinology-journals.org DOI: 10.1530/JOE-16-0276
๑) 2017 Society for Endocrinology Printed in Great Britain
Published by Bioscientifica Ltd 


\section{Changes in insulin, glucagon and somatostatin in Ngn3-Tsc1-/- mice}

We next examined the morphological and functional changes of the pancreatic islet cells during different postnatal periods. We first investigated the alteration in $\beta$-cells. Relative to wild-type animals, average size of insulin-positive $\beta$ cells increased significantly in Ngn3-TSC1-/- mice (Fig. 3A). Similarly, average size of glucagon-positive cells (Fig. 3B) and somatostatin positive $\delta$ cells (Fig. 3C) demonstrated a significant increase in Ngn3-Tsc1-/- mice. Interestingly, the number
A

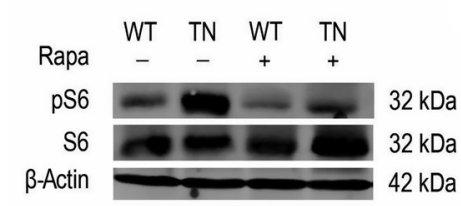

C

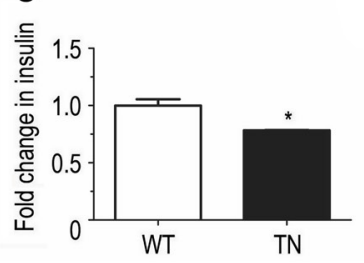

D

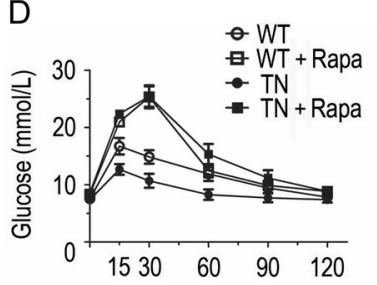

E

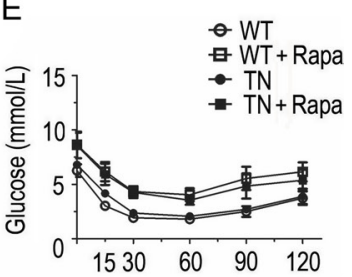

B

WT
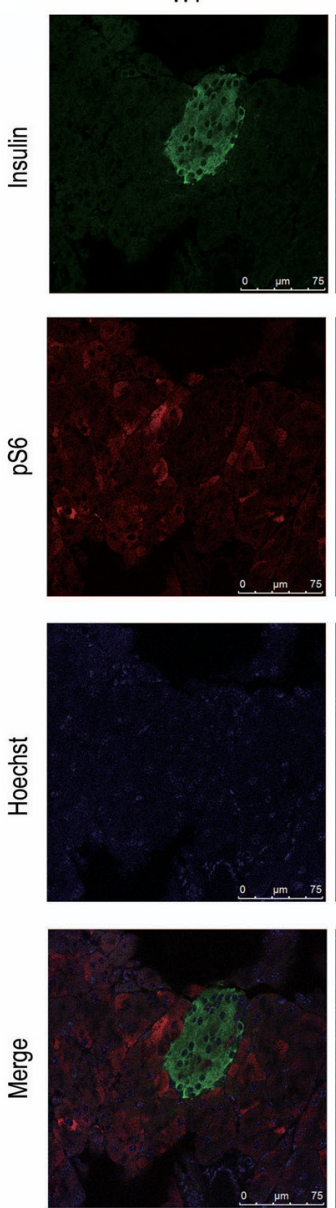

WT + Rapa
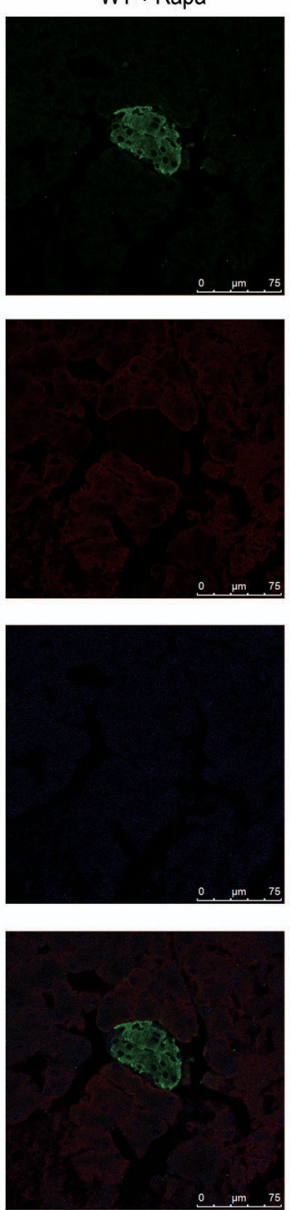

TN
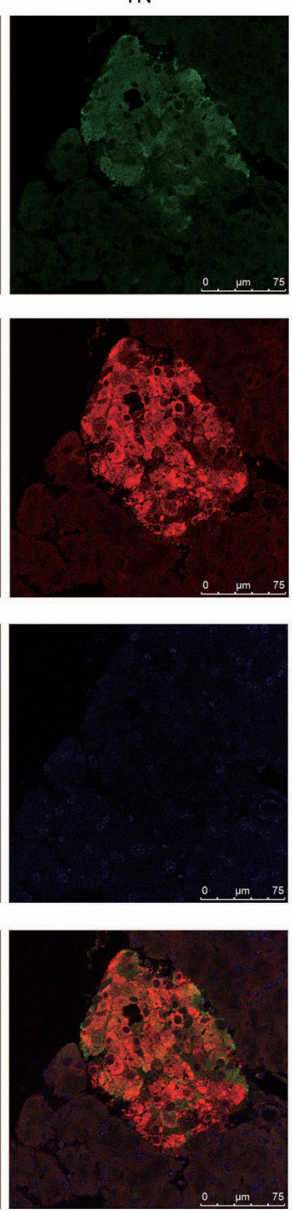

$\mathrm{TN}+\mathrm{Rapa}$
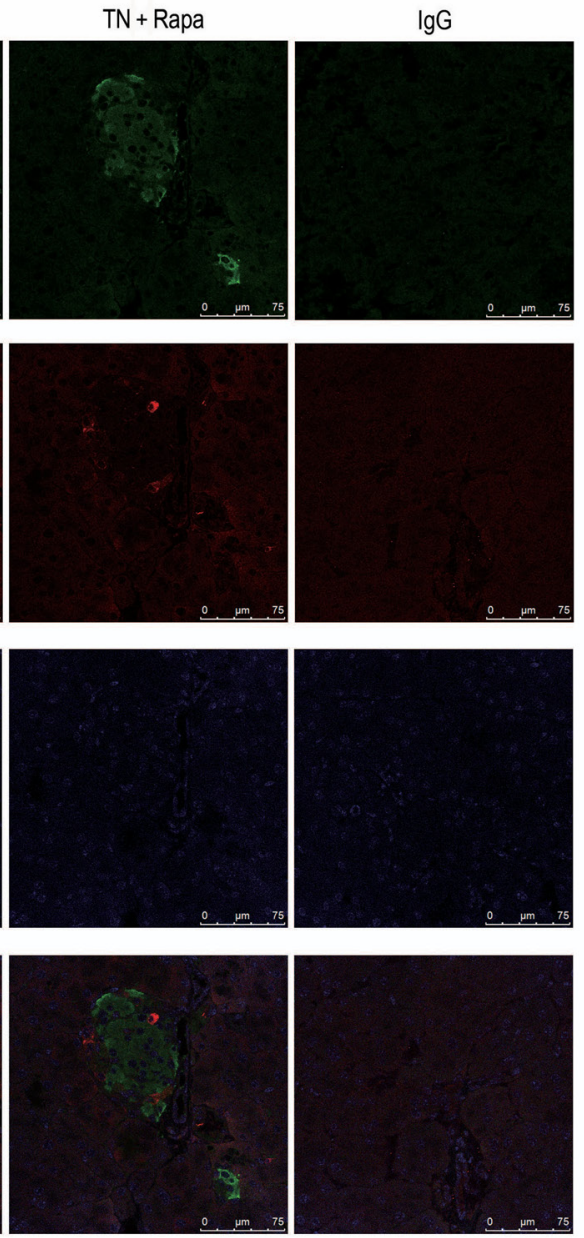

Figure 5

Rescue effect of rapamycin on $\beta$ cell hypertrophy, OGTT and ITT. (A) Representative Western blot pancreatic pS6 in wild-type (WT) and Ngn3-Tsc1-/- (TN) mice with or without rapamycin treatment were examined. S6 and $\beta$-actin were used as loading control. $n=5$ mice. (B) Co-localization of pS6 and insulin. Double-labeling of the pancreatic sections from WT and TN mice with or without rapamycin treatment were performed using pS6 (red) and insulin (green) antibodies. Nuclei were counter-stained with Hoechst 33342 fluorescent stain (blue). (C) Levels of insulin mRNA. Real-time PCR quantification of insulin mRNA from WT and TN mice after rapamycin treatment was examined and expressed as mean \pm S.E.M. (D) Oral glucose tolerance test (OGTT). Shown are the blood glucose levels of WT and TN mice treated with and without rapamycin after oral administration of glucose (2 $\mathrm{g} / \mathrm{kg}$ body weight). (E) Insulin sensitivity test (ITT). Shown are the blood glucose levels of WT and TN mice treated with or without rapamycin after intraperitoneal injection of insulin (1 IU/kg body weight). http://joe.endocrinology-journals.org
DOI: $10.1530 /$ JOE-16-0276
๑) 2017 Society for Endocrinology Printed in Great Britain
Published by Bioscientifica Ltd 
of pancreatic $\alpha$ cells decreased significantly in adult transgenic mice.

Alteration of pancreatic hormone mRNAs in Ngn3-Tsc1-/- mice was next evaluated. As shown in Fig. 3D, mRNA levels of pancreatic insulin increased significantly in both neonate and adult Ngn3-Tsc1-/mice. Interestingly, glucagon mRNA increased in neonate, but reduced markedly in adult Ngn3-Tsc1-/mice relative to the wild-type littermates (Fig. 3E). Levels of somatostatin mRNA increased significantly in both neonatal and adult Ngn3-Tsc1-/- mice compared with those of WT littermates (Fig. 3F).

Plasma levels of insulin, glucagon and somatostatin were next determined. Despite no difference in neonatal mice, plasma insulin levels increased from $1.1 \pm 0.2$ and $1.1 \pm 0.1$ in WT mice to $6.1 \pm 1.2$ and $3.6 \pm 0.6$ in Ngn3-TSC1-/- mice at the age of weaning and 16 weeks, indicating a hyperinsulinemia in Ngn3-Tsc1-/- mice (Fig. 3G). Consistent with the change in glucagon mRNA, plasma levels of glucagon demonstrated a significant increase in 4-week-old mice, followed by a marked reduction in 16-week-old mice (Fig. 3H). Unlike insulin and glucagon, there was no significant difference in the plasma somatostatin levels between Ngn3-Tsc1-/and wild-type mice at the age of neonate, weaning and 16 weeks (Fig. 3I).

\section{Alteration in glucose tolerance and insulin sensitivity in Ngn3-Tsc1-/- mice}

As insulin is crucial for glucose metabolism, we next examined the fasting blood glucose levels, oral glucose tolerance tests (OGTT) and insulin tolerance tests (ITT) in Ngn3-Tsc1-/- mice at 16 weeks old. Fasting blood glucose levels were significantly lower in the Ngn3-Tsc1-/transgenic mice $(5.2 \pm 0.2 \mathrm{mmol} / \mathrm{L})$ relative to wildtype animals $(6.4 \pm 0.1 \mathrm{mmol} / \mathrm{L})$. Further, glucose was cleared more rapidly in Ngn3-Tsc1-/- mice relative to WT littermates (Fig. 4A). This observation indicates a significant improvement in glucose tolerance in Ngn3-Tsc1-/- mice. On the other hand, there was virtually no difference in insulin sensitivity between Ngn3-TSC1-/and wild-type littermate mice (Fig. 4B). Further, the hyperglycemic clamp experiment showed a significant increase of glucose infusion rate (GIR) in the transgenic mice compared with WT littermates, indicating the enhanced insulin release stimulated by glucose (Fig. 4C).

To determine whether hyperinsulinemia is beneficial for the glucose homeostasis in obesity, we next examined the OGTT and ITT in Ngn3-Tsc1-/- mice fed HFD for
8 weeks. Unexpectedly, Ngn3-Tsc1-/- mice fed HFD demonstrated a significantly higher levels of basal blood glucose relative to wild-type littermates fed HFD (Fig. 4D, E). This alteration was accompanied with a significant impairment in glucose tolerance (Fig. 4D) and insulin sensitivity (Fig. 4E) in the transgenic mice. Relative to the transgenic mice fed NCD, the Ngn3-TSC1-/- mice fed HFD showed a blunted increase in insulin circulating levels (Fig. 4F).

\section{Rescue effects of rapamycin on $\beta$ cell hypertrophy, OGTT and ITT}

To further demonstrate that Tsc1-mTOR signaling is critical for the hypertrophy of pancreatic $\beta$-cells in Ngn3-Tsc1-/mice, we treated pregnant mice with rapamycin, a selective inhibitor for mTORC1. Rapamycin treatment blocked the increase in phosphorylation of $\mathrm{S} 6$ as determined by both Western blotting and immunostaining in Ngn3-Tsc1-/pancreatic islet cells, indicating the reversion of mTORC1 activation (Fig. 5A, B). Consistent with these observations, rapamycin blocked the upregulation of insulin mRNA in Ngn3-Tsc1-/- mice (Fig. 5C).

To test whether the improvement of glucose tolerance in Ngn3-Tsc1-/- animals is mediated by the activation of mTORC1 signaling, we performed OGTT and ITT in Ngn3-Tsc1-/- and wild-type mice treated with and without rapamycin. As shown in Fig. 5D, rapamycin impaired glucose tolerance in both groups of animals. Nonetheless, there existed no difference in OGTT between Ngng3-Tsc1-/and wild-type mice receiving rapamycin, indicating that inhibition of mTORC1 activity by rapamycin abolished the improvement of glucose tolerance in Ngng3-Tsc1-/mice (Fig. 5E). Rapamycin impaired insulin sensitivity to the same degree in both Ngn3-Tsc1-/- mice and wild-type littermates (Fig. 5F).

\section{Discussion}

\section{mTOR signaling and islet cell determination}

This study establishes mTORC1 as an important molecule in the determination of islet cells during pancreatic development. Deletion of TSC1 driven by the Ngn3 promoter activates mTORC1 signaling revealed by the detection of phosphorylation of mTOR and S6 in neonatal and adult pancreatic islet cells. Activation of mTORC1 signaling leads to a significant increase in islet mass characterized by the hypertrophy of chromogranin

Published by Bioscientifica Ltd. 
A-positive endocrine cells. All three types of islet cells including $\alpha, \beta$ and $\delta$ cells demonstrate a significant increase in cell size in the transgenic mice. These effects are likely mediated by the activation of $\mathrm{mTORC} 1$ because inhibition of mTOC1 signaling by rapamycin reverses the effect of TSC1 deletion. Furthermore, the hypertrophy of $\alpha, \beta$ and $\delta$ cells persists through the adulthood, as is the mTORC1 activity. This evidence suggests that activation of mTORC1 during embryonic period stimulates islet cell growth and the determination of all three types of islet cells. Consistently, mutation of TSC1 has been reported to cause tumors in multiple organs (Mak \& Yeung 2004). Deletion of TSC1 under the control of rat insulin promoter leads to the hypertrophy of $\beta$ cell mass and the development of prolactin-producing pituitary tumors. In addition, deletion of Pten, the upstream inhibitor of mTORC1 signaling, has been demonstrated to increase islet mass and $\beta$ cell numbers (Nguyen et al. 2006). In contrast, our data reveal that islet cell number is minimally affected in the Ngn3-Tsc1-/mice. Consistently, deletion of TSC1 under the control of rat insulin promoter demonstrates no effect on cell proliferation and number (Mori et al. 2009).

The enlarged islet cell size in the Ngn3-Tsc1-/- mice is likely resulted from the increase in protein synthesis driven by the activation of mTORC1 signaling. Indeed, our data show a significant increase in the insulin, glucagon and somatostatin mRNAs in islet cells and the relative hormone levels in plasma in the neonate and adult. Glucagon is the only exception, which shows a biphasic change: increase first in the youth mice, and then decrease during adulthood. The decrease in glucagon levels during adulthood parallels the significant increase in insulin, suggesting an interaction between these two hormones. Although we did not measure the per-islet hormone release, previous study has demonstrated that the effect of mTORC1 on insulin secretion is negligible in the Rip-Tsc1 conditional knockout mice (Mori et al. 2009).

Taken together, our findings extend the action of mTORC1 to the growth and the development of islet cells during embryonic period. Deletion of Tsc1 driven by Ngn 3 may cause an islet cell intolerance to HFD or an inability to respond appropriately. Previous studies have established mTORC1 signaling as a critical pathway to coordinate the intracellular energy levels with cell growth (Hill \& Duvillie 2000). Our study thus suggests that mTORC1 signaling may function as a critical fuel sensor to link the nutritional supply during embryonic period with the development of islet cells. This concept is supported by other studies. Reduction of dietary protein to $8 \%$ throughout gestation has been demonstrated to reduce $\beta$ cell mass and islet cell size (Van Assche et al. 1977, De Prins \& Van Assche 1982). This finding suggests that maternal environment may significantly affect the development of fetal islets. In addition, activation of mTORC1 signaling by supplementation of leucine during pregnancy has been shown to significantly decrease islet size, which is associated with increase in fetal body weight, fetal hyperglycemia and hypoinsulinemia (Rachdi et al. 2012). Further study should aim to explore how maternal nutrition affects the mTORC1 signaling to regulate islet cell determination in the developing pancreas.

\section{Selection of beta cells over alpha cells}

Another interesting finding in the present study is that the number of pancreatic $\alpha$ cells, glucagon mRNA expression and serum glucagon concentration are significantly decreased in adult Ngn3-Tsc1-/- mice despite of the transient increase in the neonatal period. Whether the decrease in the number of $\alpha$ cells and glucagon production are ascribed to the constant increase of insulin secretion by hypertrophic $\beta$ cells in the Ngn3Tsc1-/- mice remains to be determined. Previous studies have demonstrated that insulin may reduce the number of $\alpha$ cells by inhibiting their proliferation (Unger \& Orci 2010). In the streptozotocin-induced diabetic mice, apoptosis of $\beta$ cells and reduction of insulin are associated with a significant increase in the proliferation of $\alpha$ cells. Protection of $\beta$ cells from streptozotocin-induced apoptosis with dipeptidyl peptidase- 4 inhibitor reverses the mitogenic effect on alpha cells (Takeda et al. 2012). All these observations suggest that the precise interaction between these two important islet cells is crucial for the glucose homeostasis. Although our finding is consistent with these reports suggesting the potential interaction between $\alpha$ and $\beta$ cells in the islets, it is worth to note that our model of islet hyperplasia is quite different from the examples cited above. Although previous studies compare the proliferation of $\alpha$ cells in normal $\beta$ cell mass vs deficient $\beta$ cell mass, we examine that the difference between normal $\beta$ cell mass vs modestly increased $\beta$ cell mass.

\section{$\beta$ cell hypertrophy, hyperinsulinemia and glucose tolerance}

Insulin secretion relies on the normal functioning of islet $\beta$ cells. The $\beta$ cell hypertrophy induced upon

Published by Bioscientifica Ltd 
activation of mTORC1 during embryonic development of islet cells appears to result in the expansion of normal functioning islet $\beta$ cells rather than tumor formation. Consistently, improved glucose tolerance is observed in the Ngn3-Tsc1-/- mice fed NCD. Two pieces of evidence suggest that this effect is attributable to the increase of insulin release in response to glucose stimulation. First, insulin sensitivity remains unaltered. Second, glucose infusion rate increases in hyperglycemic clamp experiment. When challenged with HFD, Ngn3-Tsc1-/- mice shows hyperglycemia and derangements of glucose metabolism. The dysfunction in glucose homeostasis in these transgenic mice appears to result from the reduction in insulin secretion in response to the HFD. Although HFD induces hyperinsulinemia in wild-type mice, a significant reduction in plasma insulin is detected in Ngn3-Tsc1-/mice fed HFD relative to wild-type littermates. Further studies should explore the mechanism underlying the differential metabolic phenotypes of these transgenic mice fed chow vs HFD.

In summary, our study demonstrates that mTORC1 contributes to normal islet cell function. Further investigation should aim to determine whether mTORC1 might serve as a critical molecule in the transmission of embryonic environmental information to affect the glucose metabolism in the offspring by coordinating the maternal nutrition with the determination of pancreatic islet cells. Limitations exist for our approaches using Ngn3 promoter-driven expression of Cre gene. Ngn3 has been demonstrated to express in CNS, spinal cord and the enteroendocrine cells. In the enteroendocrine cells, alteration of mTOR signaling may affect the development of these endocrine cells, production of gut hormones and the metabolic phenotype. Further studies should aim to establish the transgenic mouse model in which mTOR signaling is manipulated only in the developing pancreatic islet cells. Unless a marker specific for pancreatic islet progenitors was identified, this approach would be challenging.

\section{Declaration of interest}

The authors declare that there is no conflict of interest that could be perceived as prejudicing the impartiality of the research reported.

\section{Funding}

This work was supported by grants from the National Natural Science Foundation of China (81330010, 81390354), and American Diabetes Association grant \#1-13-BS-225.

\section{Author contribution statement}

$L D, Y Y, L L H, Y L$ and $W Z Z$ were responsible for conception and design of the study. $L D, Y Y, L L H, J Z, Y L$ and $W Z Z$ contributed to data acquisition, analysis and interpretation. $L D, Y Y, L L H, Y L$ and $W Z Z$ contributed to data analysis and interpretation. All authors were involved in drafting the manuscript and all approved the final version. $W Z Z$ is the guarantor of this work.

\section{References}

Balcazar N, Sathyamurthy A, Elghazi L, Gould A, Weiss A, Shiojima I, Walsh K \& Bernal-Mizrachi E 2009 mTORC1 activation regulates beta-cell mass and proliferation by modulation of cyclin D2 synthesis and stability. Journal of Biological Chemistry 284 7832-7842. (doi:10.1074/jbc.M807458200)

De Prins FA \& Van Assche FA 1982 Intrauterine growth retardation and development of endocrine pancreas in the experimental rat. Biology of the Neonate 41 16-21. (doi:10.1159/000241511)

Ding L, Han L, Li Y, Zhao J, He P \& Zhang W 2014 Neurogenin3-directed cre deletion of Tsc1 gene causes pancreatic acinar carcinoma. Neoplasia 16 909-917. (doi:10.1016/j.neo.2014.08.010)

Duvillie B, Cordonnier N, Deltour L, Dandoy-Dron F, Itier JM, Monthioux E, Jami J, Joshi RL \& Bucchini D 1997 Phenotypic alterations in insulin-deficient mutant mice. PNAS 94 5137-5140. (doi:10.1073/pnas.94.10.5137)

Gradwohl G, Dierich A, LeMeur M \& Guillemot F 2000 Neurogenin3 is required for the development of the four endocrine cell lineages of the pancreas. PNAS 97 1607-1611. (doi:10.1073/pnas.97.4.1607)

Gu G, Dubauskaite J \& Melton A 2002 Direct evidence for the pancreatic lineage: NGN3+ cells are islet progenitors and are distinct from duct progenitors. Development 129 2447-2457.

Hill DJ \& Duvillie B 2000 Pancreatic development and adult diabetes. Pediatric Research 48 269-274. (doi:10.1203/00006450-20000900000002)

Holness MJ \& Sugden MC 1999 Antecedent protein restriction exacerbates development of impaired insulin action after high-fat feeding. American Journal of Physiology 276 E85-E93.

Inoki K, Zhu T \& Guan KL 2003 TSC2 mediates cellular energy response to control cell growth and survival. Cell 115 577-590. (doi:10.1016/ S0092-8674(03)00929-2)

Inoki K, Ouyang H, Zhu T, Lindvall C, Wang Y, Zhang X, Yang Q, Bennett C, Harada Y, Stankunas K, et al. 2006 TSC2 integrates Wnt and energy signals via a coordinated phosphorylation by AMPK and GSK3 to regulate cell growth. Cell 126 955-968. (doi:10.1016/j. cell.2006.06.055)

Li Y, Jiang C, Xu G, Wang N, Zhu Y, Tang C \& Wang X 2008 Homocysteine upregulates resistin production from adipocytes in vivo and in vitro. Diabetes 57 817-827. (doi:10.2337/db07-0617)

Mak BC \& Yeung RS 2004 The tuberous sclerosis complex genes in tumor development. Cancer Investigation 22 588-603. (doi:10.1081/CNV200027144)

Mori H, Inoki K, Opland D, Münzberg H, Villanueva EC, Faouzi M, Ikenoue T, Kwiatkowski DJ, Macdougald OA, Myers MG Jr, et al. 2009 Critical roles for the TSC-mTOR pathway in beta-cell function. American Journal of Physiology 297 E1013-E1022. (doi:10.1152/ ajpendo.00262.2009)

Nguyen KT, Tajmir P, Lin CH, Liadis N, Zhu XD, Eweida M, Tolasa-Karaman G, Cai F, Wang R, Kitamura T, et al. 2006 Essential role of Pten in body size determination and pancreatic beta-cell homeostasis in vivo. Molecular and Cellular Biology 26 4511-4518. (doi:10.1128/MCB.00238-06)

Rachdi L, Aiello V, Duvillie B \& Scharfmann R 2012 1-Leucine alters pancreatic beta-cell differentiation and function via the mTor signaling pathway. Diabetes 61 409-417. (doi:10.2337/db11-0765)

Published by Bioscientifica Ltd. 
Sarbassov DD, Guertin DA, Ali SM \& Sabatini DM 2005 Phosphorylation and regulation of Akt/PKB by the rictor-mTOR complex. Science $\mathbf{3 0 7}$ 1098-1101. (doi:10.1126/science.1106148)

Snoeck A, Remacle C, Reusens B \& Hoet JJ 1990 Effect of a low protein diet during pregnancy on the fetal rat endocrine pancreas. Biology of the Neonate 57 107-118. (doi:10.1159/000243170)

Takeda Y, Fujita Y, Honjo J, Yanagimachi T, Sakagami H, Takiyama Y, Makino Y, Abiko A, Kieffer TJ \& Haneda M 2012 Reduction of both beta cell death and alpha cell proliferation by dipeptidyl peptidase- 4 inhibition in a streptozotocin-induced model of diabetes in mice. Diabetologia 55 404-412. (doi:10.1007/s00125-011-2365-4)

Unger RH \& Orci L 2010 Paracrinology of islets and the paracrinopathy of diabetes. PNAS $\mathbf{1 0 7}$ 16009-16012. (doi:10.1073/pnas.1006639107)
Van Assche FA, De Prins F, Aerts L \& Verjans M 1977 The endocrine pancreas in small-for-dates infants. British Journal of Obstetrics and Gynaecology 84 751-753. (doi:10.1111/j.1471-0528.1977. tb12486.x)

Wullschleger S, Loewith R \& Hall MN 2006 TOR signaling in growth and metabolism. Cell 124 471-484. (doi:10.1016/j.cell.2006.01.016)

Xu G, Li Y, An W, Li S, Guan Y, Wang N, Tang C, Wang X, Zhu Y, Li X, et al. 2009 Gastric mammalian target of rapamycin signaling regulates ghrelin production and food intake. Endocrinology $1503637-3644$ (doi:10.1210/en.2009-0372)

Xu G, Li Z, Ding L, Tang H, Guo S, Liang H, Wang H \& Zhang W 2015 Intestinal mTOR regulates GLP-1 production in mouse L cells. Diabetologia 58 1887-1897. (doi:10.1007/s00125-015-3632-6)

Received in final form 7 October 2016

Accepted 17 October 2016

Accepted preprint published online 17 October 2016
Published by Bioscientifica Ltd. 